UTEXAS-HEP-01-22

hep-ph/0106142

\title{
$Z$ decay into two massless gauge bosons in a magnetic field
}

\author{
Todd M. Tinsleył \\ Center for Particle Physics, University of Texas, Austin, Texas, 78712, USA
}

(Dated: August 29, 2001)

\begin{abstract}
An investigation of the processes $Z \rightarrow g g$ and $Z \rightarrow \gamma \gamma$ in a background magnetic field is presented. For homogeneous fields corrections to the charged fermion propagator can be calculated in leading orders of the magnetic field. This work examines the first order contributions of the corrected propagator to decays that are otherwise zero. Results of the decay rates for varying field strengths are included.

PACS numbers: 12.20.Ds,13.38.Dg,14.70.Hp
\end{abstract}

*Electronic address: ttinsley@physics.utexas.edu 


\section{INTRODUCTION}

The interaction of matter with magnetic fields has long been studied for the important consequences it has for particle dynamics. Whether it is from the astrophysical implication of a magnetic field on the structure of a neutron star or to the Zeeman shift of energy levels within an atom, the presence of an external magnetic field can open the door to interesting effects not previously seen.

The effect of an homogeneous background field on the charged fermion propagator in QED was first studied by Schwinger [1]. Schwinger's proper-time method provides the machinery for an exact solution to the altered propagator. Recently, a weak field expansion of this method has been applied to demonstrate that there exists enhancements to neutrino-photon interactions that involve charged fermion loops [2, 3, 4. The goal of this article is to present what effects a magnetic field has on the decays of the $Z$ which are otherwise forbidden.

The Landau-Yang theorem uses Bose symmetry and rotational invariance arguments to prove that a vector particle cannot decay into two massless vector particles [5, 6]. That is, the decays $Z \rightarrow \gamma \gamma$ and $Z \rightarrow g g$ are not allowed. However we can employ the field expansion of Schwinger's method to calculate if and to what degree the magnetic field stimulates the decay.

We expand just to first order in the magnetic field $B$. This turns out to be a very good approximation considering that the relevant expansion parameter is $|Q e| B / m^{2}$, where $m$ is the mass of the fermion and $Q e$ is its charge. For the lightest of the possible fermions, the electron, the critical field turns out to be of the order $B_{0}=m_{e}^{2} / e \approx 4.4 \cdot 10^{9} \mathrm{~T}$. This field is a great deal larger than those magnetic fields produced by very dense white dwarfs, and near the neutron star magnetic field intensity. The calculation is complete to first order, but because of this expansion parameter it is shown that the dominant contributions are from the lightest quarks $(u, d)$ for $Z \rightarrow g g$ and from the electron in the $Z \rightarrow \gamma \gamma$ case.

First we briefly introduce the reader to the methodology used in determining the changes in the fermion propagator. Next, the result of that technique is presented. This result is then applied to the decay mode of the $Z$ involving two gluons. The results of this calculation are presented and contrasted with results from the similar decay into two photons. 


\section{OVERVIEW OF SCHWINGER'S RESULT}

Schwinger's method uses an integration over a proper-time variable to solve for the Green's function of the inhomogenous differential equation for a Dirac field in the presence of the electromagnetic gauge field $A_{\mu}$

$$
(i \not D-e Q \not A-m) G\left(x, x^{\prime}\right)=\delta^{4}\left(x-x^{\prime}\right) .
$$

$G\left(x, x^{\prime}\right)$ is considered the matrix element of an operator $G$, such that $G\left(x, x^{\prime}\right)=\left\langle x|G| x^{\prime}\right\rangle$. Therefore Eq. (11) is written as an operator equation with its indices suppressed

$$
(\not 1-m) G=1
$$

or

$$
\begin{aligned}
G & =\frac{1}{\not \amalg-m}=\frac{\not \Pi+m}{\not \Pi^{2}-m^{2}} \\
& =-i \int_{0}^{\infty} \mathrm{d} s(\not \Pi+m) \exp \left[i\left(\not \Pi^{2}-m^{2}\right) s\right] .
\end{aligned}
$$

$s$ is the proper-time variable, and $\Pi_{\mu}$ is a generalized momentum operator defined by

$$
\Pi_{\mu}=p_{\mu}-e Q A_{\mu}
$$

This generalized momentum operator is characterized by the following commutation properties

$$
\begin{aligned}
{\left[\Pi_{\mu}, x_{\nu}\right] } & =i g_{\mu \nu} \\
{\left[\Pi_{\mu}, \Pi_{\nu}\right] } & =-i e Q F_{\mu \nu}
\end{aligned}
$$

where $F_{\mu \nu}$ is the electromagnetic field-strength tensor.

The quantity $\exp \left[i \not \Lambda^{2} s\right]$ is regarded as an operator that evolves the system in accordance with the "Hamiltonian" $\mathcal{H}=-\not \Pi^{2}$. That is, the matrix element of the operator $U(s)=$ $\exp \left[i \not \Pi^{2} s\right]$ is the time development function that takes the state $x_{\mu}(0)=x_{\mu}^{\prime}$ to the state $x_{\mu}(s)=x_{\mu}$

$$
\left\langle x|U(s)| x^{\prime}\right\rangle=\langle x(s) \mid x(0)\rangle .
$$

This view of the transformation as an evolution of the state governed by a Hamiltonian parallels the idea that the fermion moves from one space-time location to another in a way 
that is determined by this Hamiltonian. Accordingly, there exist "equations of motion" satisfied by the generalized position and momentum operators

$$
\begin{aligned}
\frac{\mathrm{d} x_{\mu}}{\mathrm{d} s} & =-i\left[x_{\mu}, \mathcal{H}\right]=2 \Pi_{\mu} \\
\frac{\mathrm{d} \Pi_{\mu}}{\mathrm{d} s} & =-i\left[\Pi_{\mu}, \mathcal{H}\right]=2 Q e F_{\mu \nu} \Pi^{\nu} .
\end{aligned}
$$

The relationship in Eq. (7b) only holds when $F_{\mu \nu}$ is a constant. This is precisely the case in which we are concerned. For calculation purposes the magnetic field is considered to be constant and directed along the $z$-axis $\left(F_{12}=-F_{21}=B\right.$, and $F_{i j}=0$ for $\left.i, j \neq 1,2\right)$. In addition there are three differential equations that describe the transformation function

$$
\begin{aligned}
i \partial_{s}\langle x(s) \mid x(0)\rangle & =\langle x(s)|\mathcal{H}| x(0)\rangle \\
\left(i \partial_{\mu}+e Q A_{\mu}\right)\langle x(s) \mid x(0)\rangle & =\left\langle x(s)\left|\Pi_{\mu}(s)\right| x(0)\right\rangle \\
\left(-i \partial_{\mu}^{\prime}+e Q A_{\mu}\right)\langle x(s) \mid x(0)\rangle & =\left\langle x(s)\left|\Pi_{\mu}(0)\right| x(0)\right\rangle,
\end{aligned}
$$

and a boundary condition

$$
\left.\langle x(s) \mid x(0)\rangle\right|_{s \rightarrow 0}=\delta^{4}\left(x-x^{\prime}\right) .
$$

Using Eqs. (17), (8), and (9) one can solve for the explicit form of the Green's function. For the purposes of this article, only the result will be given [10]. Choosing a straight line to connect the space-time points $x$ and $x^{\prime}$, the Green's function is

$$
G\left(x, x^{\prime}\right)=\Phi\left(x, x^{\prime}\right) \int \frac{\mathrm{d}^{4} p}{(2 \pi)^{4}} e^{-i p\left(x-x^{\prime}\right)} \mathcal{G}(p),
$$

with

$$
\Phi\left(x, x^{\prime}\right)=\exp \left[-i e Q \int_{x^{\prime}}^{x} \mathrm{~d} \xi^{\mu} A_{\mu}(\xi)\right]
$$

and

$$
\begin{aligned}
\mathcal{G}(p)= & -i \int_{0}^{\infty} \mathrm{d} s \exp \left[-i s\left(m^{2}-p_{\|}^{2}+\frac{\tan (e Q B s)}{e Q B s} p_{\perp}^{2}\right)\right] \\
& \times\left[e^{i e Q B s \sigma_{3}} \frac{\left(m+\not p_{\|}\right)}{\cos (e Q B s)}-\frac{\not{ }_{\perp}}{\cos ^{2}(e Q B s)}\right] .
\end{aligned}
$$

The quantity $p_{\|}^{2}$ indicates $p_{0}^{2}-p_{3}^{2}$, while $p_{\perp}^{2}=p_{1}^{2}+p_{2}^{2}$. Similarly, $\not \not_{\|}=\gamma_{0} p_{0}-\gamma_{3} p_{3}$ and $\not p_{\perp}=\gamma_{1} p_{1}+\gamma_{2} p_{2}$. By expanding Eq. (12) to first order in the magnetic field the integration 
simplifies, and our result is

$$
\mathcal{G}(p) \approx(\not p-m)^{-1}-\frac{i e Q}{2} F_{\alpha \beta} \frac{\gamma^{\alpha} \gamma^{\beta}\left(\not \|_{\|}+m\right)}{\left(p^{2}-m^{2}\right)^{2}}
$$

By making use of the fact that the magnetic field is homogeneous, the result in Eq. (13) was cast into covariant notation.

\section{APPLICATION TO $Z \rightarrow g g$}

At this point the general results from the previous section are used to calculate the decay rate of the neutral $Z$ boson into to two gluons. To lowest order in the perturbation theory there are two Feynman diagrams which contribute to this process; see Fig. 11. Let us first turn our attention to the leftmost contribution of Fig. 1.

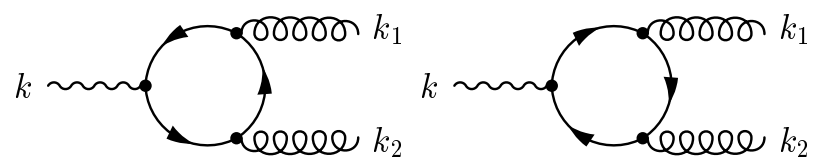

FIG. 1: The contributions to the decay of the $Z$ with momentum $k$ into two gluons with momenta $k_{1}$ and $k_{2}$.

The T-Matrix for the first of our diagrams is written as

$$
\begin{aligned}
T_{A}= & \sum_{i} \sum_{a, b} \frac{\pi^{3 / 2} \alpha_{s} \sqrt{\alpha}}{\cos \theta_{W} \sin \theta_{W}} \operatorname{tr}\left(\lambda^{a} \lambda^{b}\right) \int \mathrm{d}^{4} x \int \mathrm{d}^{4} y \int \mathrm{d}^{4} z \\
& \times \operatorname{tr}\left[\gamma^{\mu} G^{i}(x, y) \gamma^{\nu} G^{i}(y, z) \gamma^{\sigma}\left(g_{V}^{i}-g_{A}^{i} \gamma^{5}\right) G^{i}(z, x)\right] \\
& \times \epsilon_{\mu}^{* a}\left(k_{1}\right) \epsilon_{\nu}^{* b}\left(k_{2}\right) \epsilon_{\sigma}(k) \exp \left[-i\left(k z-k_{1} x-k_{2} y\right)\right] .
\end{aligned}
$$

The sum over $i$ indicates the sum over all possible fermions that contribute to this loop diagram. The sums over $a$ and $b$ indicate the sums over the possible states of the gluons. However, one of these sums is eliminated by virtue of the trace over the two $\mathrm{SU}(3)$ generators $\operatorname{tr}\left(\lambda^{a} \lambda^{b}\right)=2 \delta^{a b}$. From this point the summation over color will be represented by the

repeated color index $a$. The couplings $g_{V}^{i}$ and $g_{A}^{i}$ are the vector and axial vector couplings, respectively; both are dependent on the particular fermion that couples to the $Z$ boson. $\alpha_{s}$ is the strong coupling constant evaluated at $M_{Z}$.

Because there is the combination of the three Green's functions in Eq. (14), there exists a product of three phases of the same form as in Eq. (11). As indicated previously, the 
integration from two space-time points is along a straight line. A convenient choice of gauge for this integration is one in which

$$
A(\xi)=\frac{B}{2}\left(0, z_{2}-\xi_{2}, \xi_{1}-z_{1}, 0\right) .
$$

Generally, the zeroth and third components of $A_{\mu}$ can be any constant but the above is chosen for its simplicity. With the choice of gauge found in Eq. (15) the total phase factor of the diagram is written

$$
\begin{aligned}
\Phi(x, y) \Phi(y, z) \Phi(z, x) & =\exp \left[\frac{i e Q}{2}(z-y)^{\mu} F_{\mu \nu}(z-x)^{\nu}\right] \\
& \approx 1+\frac{i e Q}{2}(z-y)^{\mu} F_{\mu \nu}(z-x)^{\nu} .
\end{aligned}
$$

The fact that the magnetic field is constant and along the $z$-axis has been exploited. When the first order corrections to the Green's function found in Eqs. (13) and (16) are substituted into Eq. (14), the result is thought of as the sum of five separate contributions

$$
T_{A}=T_{A 0}+T_{A \mathrm{pr} 1}+T_{A \mathrm{pr} 2}+T_{A \mathrm{pr} 3}+T_{A \mathrm{ph}}
$$

There is the zeroth order contribution to the diagram $T_{A 0}$ which is completely independent of the magnetic field. There is a first order correction to each of the legs of the fermion loop $T_{A \mathrm{pr} 1}, T_{A \mathrm{pr} 2}$ and $T_{A \mathrm{pr} 3}$. And lastly, there is a first order correction to the overall phase of the diagram $T_{A \mathrm{ph}}$. Because the invariant amplitude of a given diagram $\mathcal{M}$ is defined through the relationship $T=(2 \pi)^{4} \mathcal{M} \delta^{4}\left(k-k_{1}-k_{2}\right)$, it is straightforward to show that the zeroth order contribution from the first diagram is

$$
\begin{aligned}
\mathcal{M}_{A 0}= & \frac{2 \pi^{3 / 2} \alpha_{s} \sqrt{\alpha}}{\cos \theta_{W} \sin \theta_{W}} \sum_{i} \sum_{a} \epsilon_{\mu}^{* a}\left(k_{1}\right) \epsilon_{\nu}^{* a}\left(k_{2}\right) \epsilon_{\sigma}(k) \\
& \times \int \frac{\mathrm{d}^{4} p}{(2 \pi)^{4}} \operatorname{tr}\left[\gamma^{\mu}(\not p-m)^{-1} \gamma^{\nu}\left(\not p+\not k_{2}-m\right)^{-1}\right. \\
& \left.\times \gamma^{\sigma}\left(g_{V}^{i}-g_{A}^{i} \gamma^{5}\right)\left(\not p-\not k_{1}-m\right)^{-1}\right] .
\end{aligned}
$$

For the first order correction to one of the legs of the loop, the contribution to the amplitude is

$$
\begin{aligned}
\mathcal{M}_{A \mathrm{pr} 1}= & \frac{-i \pi^{3 / 2} e F_{\alpha \beta} \alpha_{s} \sqrt{\alpha}}{\cos \theta_{W} \sin \theta_{W}} \sum_{i} \sum_{a} Q \epsilon_{\mu}^{* a}\left(k_{1}\right) \epsilon_{\nu}^{* a}\left(k_{2}\right) \epsilon_{\sigma}(k) \\
& \times \int \frac{\mathrm{d}^{4} p}{(2 \pi)^{4}} \operatorname{tr}\left[\gamma^{\mu} \frac{\gamma^{\alpha} \gamma^{\beta}(\not p+m)}{\left(p^{2}-m^{2}\right)^{2}} \gamma^{\nu}\left(\not p+\not k_{2}-m\right)^{-1}\right. \\
& \left.\times \gamma^{\sigma}\left(g_{V}^{i}-g_{A}^{i} \gamma^{5}\right)\left(\not p-\not k_{1}-m\right)^{-1}\right] .
\end{aligned}
$$


The corrections to the remaining legs of the fermion loop are similar to Eq. (19). However, for the contribution due to the phase it is necessary we recognize that

$$
\begin{aligned}
T_{A \mathrm{ph}} \propto & (z-y)^{\mu}(z-x)^{\nu} e^{-i p^{\prime}(y-z)} e^{-i p^{\prime \prime}(z-x)} \\
& \times \operatorname{tr}\left[\gamma^{\mu}(\not p-m)^{-1} \gamma^{\nu}\left(\not p^{\prime}-m\right)^{-1} \gamma^{\sigma}\left(g_{V}^{i}-g_{A}^{i} \gamma^{5}\right)\left(\not p^{\prime \prime}-m\right)^{-1}\right]
\end{aligned}
$$

where $p^{\prime}$ is the momentum carried by the fermion as it moves from point $z$ to point $y$, and $p^{\prime \prime}$ is its momentum from point $x$ to $z$. The relationship in Eq. (20) is rewritten in terms of derivatives with respect to the fermion momenta

$$
\begin{aligned}
T_{A \mathrm{ph}} \propto & \left(\frac{\partial}{\partial p_{\mu}^{\prime}} \frac{\partial}{\partial p_{\nu}^{\prime \prime}} e^{-i p^{\prime}(y-z)} e^{-i p^{\prime \prime}(z-x)}\right) \\
& \times \operatorname{tr}\left[\gamma^{\mu}(\not p-m)^{-1} \gamma^{\nu}\left(\not p^{\prime}-m\right)^{-1} \gamma^{\sigma}\left(g_{V}^{i}-g_{A}^{i} \gamma^{5}\right)\left(\not \not^{\prime \prime}-m\right)^{-1}\right]
\end{aligned}
$$

By making use of integration by parts and the identity

$$
\frac{\partial}{\partial x} \mathcal{C}^{-1}(x)=-\mathcal{C}^{-1}(x) \frac{\partial \mathcal{C}(x)}{\partial x} \mathcal{C}^{-1}(x),
$$

the first order contribution of the correction to the phase to the amplitude is

$$
\begin{aligned}
\mathcal{M}_{A \mathrm{ph}}= & \frac{-i \pi^{3 / 2} e F_{\alpha \beta} \alpha_{s} \sqrt{\alpha}}{\cos \theta_{W} \sin \theta_{W}} \sum_{i} \sum_{a} Q \epsilon_{\mu}^{* a}\left(k_{1}\right) \epsilon_{\nu}^{* a}\left(k_{2}\right) \epsilon_{\sigma}(k) \\
& \times \int \frac{\mathrm{d}^{4} p}{(2 \pi)^{4}} \operatorname{tr}\left[\gamma^{\mu}(\not p-m)^{-1} \gamma^{\nu}\left(\not p+\not k_{2}-m\right)^{-1} \gamma^{\beta}\left(\not p+\not k_{2}-m\right)^{-1}\right. \\
& \left.\times \gamma^{\sigma}\left(g_{V}^{i}-g_{A}^{i} \gamma^{5}\right)\left(\not p-\not k_{1}-m\right)^{-1} \gamma^{\alpha}\left(\not p-\not k_{1}-m\right)^{-1}\right] .
\end{aligned}
$$

We follow the same procedure for the second diagram of Fig. 1 as we did for the first. The results obtained are similar to those found in Eqs. (18), (19), and (22) with only the labels $(1,2)$ interchanged.

\section{THE DECAY RATE}

The sum of the zeroth order contributions from the two diagrams vanishes. This is consistent with the fact that the process $Z \rightarrow g g$ is forbidden by the Landau-Yang Theorem in the absence of a magnetic field [5, 6]. The use of the charge conjugation operator simplifies the contributions that are first order in the magnetic field. We find that the axial vector parts of the two diagrams cancel while the vector parts are identical and add. Tracing over 
the Dirac indices and integrating over the loop momentum results in an amplitude that is proportional to forty-four separate tensor structures formed by possible contractions of the polarization vectors, the field-strength tensor, and the gluon momenta. Since the amplitude is symmetric under interchange of the labels $(1,2)$, we find that the tensor structures can be grouped into twenty-two pairs; each pair having its own unitless coefficient which depends on the possible fermion masses, charges, and couplings in the charge loop. Limiting the gluons to be only transversely polarized allows us to drop all terms proportional to $\left(\epsilon_{i}^{* a} \cdot k_{i}\right)$. This cuts the number of tensor structures in half to eleven. Thus, the amplitude is written as

$$
\begin{aligned}
\mathcal{M}_{Z \rightarrow g g}= & \left(\frac{e \alpha_{s} \sqrt{\alpha}}{32 \sqrt{\pi} M_{Z} \sin \theta_{W} \cos \theta_{W}}\right) \times\left\{N_{1}\left(\epsilon \cdot\left(k_{1}-k_{2}\right)\right)\left(\epsilon_{1}^{* a} F \epsilon_{2}^{* a}\right)\left(1 / M_{Z}\right)\right. \\
& +N_{2}\left[\left(\epsilon_{1}^{* a} \cdot \epsilon\right)\left(\epsilon_{2}^{* a} F k_{1}\right)+\left(\epsilon_{2}^{* a} \cdot \epsilon\right)\left(\epsilon_{1}^{* a} F k_{2}\right)\right]\left(1 / M_{Z}\right) \\
& +N_{3}\left[\left(\epsilon_{1}^{* a} \cdot \epsilon\right)\left(\epsilon_{2}^{* a} F k_{2}\right)+\left(\epsilon_{2}^{* a} \cdot \epsilon\right)\left(\epsilon_{1}^{* a} F k_{1}\right)\right]\left(1 / M_{Z}\right) \\
& +N_{4}\left(\epsilon_{1}^{* a} \cdot \epsilon_{2}^{* a}\right)\left(\epsilon F\left(k_{1}+k_{2}\right)\right)\left(1 / M_{Z}\right) \\
& +N_{5}\left(\epsilon_{1}^{* a} \cdot \epsilon_{2}^{* a}\right)\left(\epsilon \cdot\left(k_{1}-k_{2}\right)\right)\left(k_{1} F k_{2}\right)\left(1 / M_{Z}\right)^{3} \\
& +N_{6}\left(\epsilon_{1}^{* a} \cdot k_{2}\right)\left(\epsilon_{2}^{* a} \cdot k_{1}\right)\left(\epsilon \cdot\left(k_{1}-k_{2}\right)\right)\left(k_{1} F k_{2}\right)\left(1 / M_{Z}\right)^{5} \\
& +N_{7}\left[\left(\epsilon_{1}^{* a} \cdot \epsilon\right)\left(\epsilon_{2}^{* a} \cdot k_{1}\right)-\left(\epsilon_{2}^{* a} \cdot \epsilon\right)\left(\epsilon_{1}^{* a} \cdot k_{2}\right)\right]\left(k_{1} F k_{2}\right)\left(1 / M_{Z}\right)^{3} \\
& +N_{8}\left[\left(\epsilon_{2}^{* a} \cdot k_{1}\right)\left(\epsilon \cdot k_{2}\right)\left(\epsilon_{1}^{* a} F k_{2}\right)+\left(\epsilon_{1}^{* a} \cdot k_{2}\right)\left(\epsilon \cdot k_{1}\right)\left(\epsilon_{2}^{* a} F k_{1}\right)\right]\left(1 / M_{Z}\right)^{3} \\
& +N_{9}\left[\left(\epsilon_{2}^{* a} \cdot k_{1}\right)\left(\epsilon \cdot k_{1}\right)\left(\epsilon_{1}^{* a} F k_{2}\right)+\left(\epsilon_{1}^{* a} \cdot k_{2}\right)\left(\epsilon \cdot k_{2}\right)\left(\epsilon_{2}^{* a} F k_{1}\right)\right]\left(1 / M_{Z}\right)^{3} \\
& +N_{10}\left[\left(\epsilon_{2}^{* a} \cdot k_{1}\right)\left(\epsilon \cdot k_{2}\right)\left(\epsilon_{1}^{* a} F k_{1}\right)+\left(\epsilon_{1}^{* a} \cdot k_{2}\right)\left(\epsilon \cdot k_{1}\right)\left(\epsilon_{2}^{* a} F k_{2}\right)\right]\left(1 / M_{Z}\right)^{3} \\
& \left.+N_{11}\left(\epsilon_{1}^{* a} \cdot k_{2}\right)\left(\epsilon_{2}^{* a} \cdot k_{1}\right)\left(\epsilon F\left(k_{1}+k_{2}\right)\right)\left(1 / M_{Z}\right)^{3}\right\}
\end{aligned}
$$

where $M_{Z}$ is the mass of the $Z, \theta_{W}$ is the Weinberg angle, and the $N_{i}$ are the unitless coefficients. The reader should see the appendix for a detailed explanation of how the coefficients are defined.

At this point we confirm that our amplitude is gauge invariant by replacing a polarization vector by its associated momentum

$$
\epsilon_{i}^{* \mu}\left(k_{i}\right) \rightarrow k_{i}^{\mu}
$$

and verifying that the amplitude vanishes. For these purposes we drop the color index $a$. The gauge invariance implies six independent relations among the coefficients that reduce 
the number of independent coefficients from eleven to five. These six relations and five coefficients are given explicitly in Eqs. (A1) and (A2) of the appendix.

What is of real interest, however, is the square of this amplitude. When the square is taken and evaluated in the center of mass frame of the $Z$, we find that the square of the amplitude depends on only three of our five independent tensor coefficients

$$
\left|\mathcal{M}_{Z \rightarrow g g}\right|^{2}=\frac{e^{2} \alpha \alpha_{s}^{2} B^{2}}{256 \pi M_{Z}^{2} \cos ^{2} \theta_{W} \sin ^{2} \theta_{W}}\left(4\left|N_{1}\right|^{2} \cos ^{2} \theta+\left|N_{2}-N_{3}\right|^{2} \sin ^{2} \theta\right),
$$

where $\theta$ is the polar angle as measured from the direction in which the magnetic field points.

It is useful to extract from Eq. (25) the effective expansion parameter for the magnetic field. That is, it was discussed earlier that the perturbation theory for the magnetic field is an expansion in $|Q e| B / m^{2}$. Because this is fermion dependent, it is useful to pick the lightest fermion and make $B / B_{0}$ the expansion parameter. Recall that $B_{0}=m_{e}^{2} / e$. Making this substitution, Eq. (25) becomes

$$
\left|\mathcal{M}_{Z \rightarrow g g}\right|^{2}=\frac{\alpha \alpha_{s}^{2} M_{Z}^{2}\left(B / B_{0}\right)^{2}}{256 \pi \cos ^{2} \theta_{W} \sin ^{2} \theta_{W}}\left(\frac{m_{e}}{M_{Z}}\right)^{4}\left(4\left|N_{1}\right|^{2} \cos ^{2} \theta+\left|N_{2}-N_{3}\right|^{2} \sin ^{2} \theta\right) \text {. }
$$

At this point it is instructive to note that the leading contributions of $N_{1}$ go as $\ln \left(\frac{M_{Z}}{m}\right)^{2}$, while for both $N_{2}$ and $N_{3}$ the leading contributions go as $\left(\frac{M_{Z}}{m}\right)^{2}$ [11]. Qualitatively, this means that only the lightest of the fermions make significant contributions to the the decay rate. It also means that the $Z$ is many times more likely to decay such that the gluons travel perpendicular to the magnetic field rather than parallel. Computation reveals that this factor is

$$
2 \cdot 10^{13} \gtrsim \frac{\left|N_{2}-N_{3}\right|^{2}}{4\left|N_{1}\right|^{2}} \gtrsim 6 \cdot 10^{10}
$$

The large range in values is a symptom of the very large uncertainty in the current quark masses. If the constituent quark masses are used, the ratio drops to a factor of $2 \cdot 10^{4}$.

The decay rate for this process is

$$
\Gamma_{Z \rightarrow g g}=\frac{\alpha \alpha_{s}^{2} M_{Z}\left(B / B_{0}\right)^{2}}{3 \cdot 2^{11} \pi^{2} \cos ^{2} \theta_{W} \sin ^{2} \theta w}\left(\frac{m_{e}}{M_{Z}}\right)^{4}\left(2\left|N_{1}\right|^{2}+\left|N_{2}-N_{3}\right|^{2}\right) .
$$

Because of the large current quark mass ranges quoted by the Particle Data Group [7], there is a corresponding large range in possible values for the decay rate. We choose to use the mean values of these masses for computation. Seperate calculations are carried out using 
the constituent quark masses. Given below are the results of the decay rate for both the current and constituent mass calculations [12], respectively,

$$
\Gamma_{Z \rightarrow g g}=\left\{\begin{array}{c}
5.10 \cdot 10^{-7} \\
9.60 \cdot 10^{-15}
\end{array}\right\}\left(\frac{B}{B_{0}}\right)^{2} \mathrm{MeV} .
$$

The values of the branching ratio of the above decay rate with the total width of the $Z$ in the absence of a magnetic field are

$$
\frac{\Gamma_{Z \rightarrow g g}}{\Gamma}=\left\{\begin{array}{l}
2.04 \cdot 10^{-10} \\
3.85 \cdot 10^{-18}
\end{array}\right\}\left(\frac{B}{B_{0}}\right)^{2} .
$$

For comparison purposes it is convenient to contrast the results of the two gluon case with the two photon case. The results of the latter decay were determined in a manner completely analogous to that which has been outlined in this article for the decay $Z \rightarrow g g$. The values of the decay rate for the process $Z \rightarrow \gamma \gamma$ are

$$
\Gamma_{Z \rightarrow \gamma \gamma}=\left\{\begin{array}{l}
8.33 \cdot 10^{-8} \\
6.63 \cdot 10^{-8}
\end{array}\right\}\left(\frac{B}{B_{0}}\right)^{2} \mathrm{MeV}
$$

Notice that the degree to which the two values differ is much smaller than in the two gluon case. This is because the major contribution to the process is due to the fermion mass being that of the electron, which is known very well. The corresponding values for the branching ratio are

$$
\frac{\Gamma_{Z \rightarrow \gamma \gamma}}{\Gamma}=\left\{\begin{array}{l}
3.34 \cdot 10^{-11} \\
2.66 \cdot 10^{-11}
\end{array}\right\}\left(\frac{B}{B_{0}}\right)^{2} .
$$

\section{CONCLUSION}

This article has demonstrated that the existence of a magnetic field can stimulate decays that were otherwise forbidden. Schwinger's powerful proper-time method was used in conjunction with a first order expansion in the field to determine what corrections exist for the Dirac Green's function. These new contributions were analyzed in the specific decays $Z \rightarrow g g$ and $Z \rightarrow \gamma \gamma$. Though the resulting decay rates are small, they are non-zero. In addition it was also found that there exists a very large asymmetry as to whether the decay products will exit perpendicular or parallel to the magnetic field. 


\section{APPENDIX A}

As noted in the body of the article, the amplitude for the decay $Z \rightarrow g g$ to first order in an external magnetic field (Fig. 1) is the sum the contributions from the zeroth order amplitude (Eq. (18)), first order corrections to each leg of the charged ferion loop (Eq. (19) and the like), the first order correction to the phase of the amplitude (Eq. (22)), and similar corrections for the crossed process which one obtains by interchange of the labels $(1,2)$.

Evaluation of this amplitude gives the result found in Eq. (23). Gauge invariance of this amplitude implies the following six relationships which we confirmed,

$$
\begin{aligned}
& N_{6}=4 N_{1}-4 N_{2}-2 N_{5} \\
& N_{7}=2 N_{2} \\
& N_{8}=2 N_{1}-2 N_{2} \\
& N_{9}=-2 N_{1} \\
& N_{10}=-2 N_{3} \\
& N_{11}=-2 N_{4} .
\end{aligned}
$$


Explicitly, the five remaining independent coefficients are

$$
\begin{aligned}
& N_{1}=\sum_{i} \frac{Q_{i} g_{v}^{i}}{\left(t_{i}-4\right)^{2}} \times\left[\left(-128 t_{i}^{2}+768 t_{i}-1024\right)\right. \\
& +\left(96 t_{i}^{2}-896 t_{i}+2048\right) A\left(t_{i}\right) \\
& \left.-\left(64 t_{i}-512+1024 t_{i}^{-1}\right) B\left(t_{i}\right)\right] \\
& N_{2}=\sum_{i} \frac{Q_{i} g_{v}^{i}}{\left(t_{i}-4\right)^{2}} \times\left[\left(-8 t_{i}^{3}+32 t_{i}^{2}-256 t_{i}+1024\right)\right. \\
& -\left(80 t_{i}^{2}-448 t_{i}+512\right) A\left(t_{i}\right) \\
& \left.+\left(16 t_{i}^{2}-64 t_{i}-256+1024 t_{i}^{-1}\right) B\left(t_{i}\right)\right] \\
& N_{3}=\sum_{i} \frac{Q_{i} g_{v}^{i}}{\left(t_{i}-4\right)^{2}} \times\left[\left(-(8 / 3) t_{i}^{3}+(160 / 3) t_{i}^{2}-(512 / 3) t_{i}\right)\right. \\
& +\left(48 t_{i}^{2}-320 t_{i}+512\right) A\left(t_{i}\right) \\
& \left.-\left(16 t_{i}^{2}-128 t_{i}+256\right) B\left(t_{i}\right)\right] \\
& N_{4}=\sum_{i} \frac{Q_{i} g_{v}^{i}}{\left(t_{i}-4\right)^{2}} \times\left[\left(8 t_{i}^{3}-64 t_{i}^{2}+128 t_{i}\right)\right. \\
& -\left(48 t_{i}^{2}-384 t_{i}+768\right) A\left(t_{i}\right) \\
& \left.+\left(16 t_{i}^{2}-128 t_{i}+256\right) B\left(t_{i}\right)\right] \\
& N_{5}=\sum_{i} \frac{Q_{i} g_{v}^{i}}{\left(t_{i}-4\right)^{2}} \times\left[\left(16 t_{i}^{3}+64 t_{i}^{2}-1024 t_{i}+2048\right)\right. \\
& -\left(224 t_{i}^{2}-1920 t_{i}+4096\right) A\left(t_{i}\right) \\
& \left.+\left(32 t_{i}^{2}-128 t_{i}-512+2048 t_{i}^{-1}\right) B\left(t_{i}\right)\right],
\end{aligned}
$$

where $t_{i}$ is defined by the square of the ratio of the $Z$ mass to the quark mass

$$
t_{i}=\left(\frac{M_{Z}}{m_{i}}\right)^{2},
$$

and the functions $A\left(t_{i}\right)$ and $B\left(t_{i}\right)$ are defined by [8]

$$
\begin{array}{rlrl}
A(t)=\int_{0}^{1} \mathrm{~d} x \ln [1-t x(1-x)-i \delta] & =2\left[\sqrt{\frac{t-4}{t}} \sinh ^{-1} \sqrt{\frac{-t}{4}}-1\right], & & \text { for } t \leq 0 \\
& =2\left[\sqrt{\frac{4-t}{t}} \sin ^{-1} \sqrt{\frac{t}{4}}-1\right], & & \text { for } 4 \geq t \geq 0 \\
& =2\left[\sqrt{\frac{t-4}{t}}\left(\cosh ^{-1} \sqrt{\frac{t}{4}}-\frac{i \pi}{2}\right)-1\right], \text { for } t \geq 4,
\end{array}
$$


and

$$
\begin{array}{rlrl}
B(t)=\int_{0}^{1} \mathrm{~d} x \frac{\ln [1-t x(1-x)-i \delta]}{x(1-x)} & =4\left[\sinh ^{-1} \sqrt{\frac{-t}{4}}\right]^{2}, & & \text { for } t \leq 0 \\
& =-4\left[\sin ^{-1} \sqrt{\frac{t}{4}}\right]^{2}, & \text { for } 4 \geq t \geq 0 \\
& =4\left[\cosh ^{-1} \sqrt{\frac{t}{4}}\right]^{2}-\pi^{2}-4 i \pi \cosh ^{-1} \sqrt{\frac{t}{4}}, & \text { for } t \geq 4 .
\end{array}
$$

Notice that in Eqs. (A2) there is a sum over all possible quarks in the charge loop. For

the decay $Z \rightarrow \gamma \gamma$ this could also include the electron. $Q_{i}$ and $g_{v}^{i}$ are the charge and the associated vector coupling of the fermion, repectively. The factors of $M_{Z}$ present are due to the evaluation of the dot product between the two gluon momenta

$$
\begin{aligned}
k_{1} \cdot k_{2} & =\left(k_{1}+k_{2}\right)^{2} / 2 \\
& =k^{2} / 2 \\
& =M_{Z}^{2} / 2,
\end{aligned}
$$

where $k$ is the four-momentum of the $Z$.

\section{ACKNOWLEDGMENTS}

I wish to extend special thanks to Duane Dicus for the useful discussions and help given throughout the course of this work.

[1] J. Schwinger, Phys. Rev. 82, 664 (1951).

[2] T.-K. Chyi, C.-W. Hwang, W. F. Kao, G.-L. Lin, K.-W. Ng, and J.-J. Tseng, Phys. Lett. B 466, 274 (1999), hep-ph/9907384.

[3] D. A. Dicus and W. W. Repko, Phys. Lett. B 482, 141 (2000), hep-ph/0003305.

[4] T.-K. Chyi, C.-W. Hwang, W. F. Kao, G.-L. Lin, K.-W. Ng, and J.-J. Tseng, Phys. Rev. D 62, 105014 (2000), hep-th/9912134.

[5] L. D. Landau, Dokl. Akad. Nauk. USSR 60, 242 (1948).

[6] C. N. Yang, Phys. Rev. 77, 242 (1950). 
[7] D. Groom, M. Aguilar-Benitez, C. Amsler, R. Barnett, P. Burchat, C. Carone, C. Caso, G. Conforto, O. Dahl, M. Doser, S. Eidelman, J. Feng, et al., The European Physical Journal C15, 1+ (2000), URL http://pdg.lbl.gov.

[8] Y. Shima, Phys. Rev. 142, 944 (1966).

[9] D. H. Perkins, Introduction to High Energy Physics (Addison-Wesley Publishing company, Inc., Massachusetts 01867, USA, 1982).

[10] For an excellent treatment of this solution see Ref. [4] or Schwinger's own work in Ref. [1].

[11] One can obtain this result by expanding the relationships in Eqs. (A2b), (A2d), (A2d), (A4), and (A5) of the appendix for large values of $\left(\frac{M_{Z}}{m}\right)^{2}$.

[12] The current quark masses that are used in this calculation are: $m_{u}=3 \mathrm{MeV}, m_{d}=6 \mathrm{MeV}$, $m_{s}=122.5 \mathrm{MeV}, m_{c}=1.25 \mathrm{GeV}, m_{b}=4.2 \mathrm{GeV}$, and $m_{t}=174.3 \mathrm{GeV}$. The constituent quark mass values used are: $m_{u}=m_{d}=350 \mathrm{MeV}, m_{s}=550 \mathrm{MeV}, m_{c}=1.8 \mathrm{GeV}, m_{b}=4.5 \mathrm{GeV}$, and $m_{t}=174.3 \mathrm{GeV}$ [9]. 